\title{
Design of Control System in Continuous Casting Machine Based on PLC
}

\author{
Guojie Song ${ }^{1}$
}

${ }^{1}$ Siping Professional College, Siping, Jilin, 136002,

\author{
KEYWORDS: Continuous Casting Machine; PLC; Automatic Control
}

\begin{abstract}
The quality of slab continuous casting process control and technical level has a close relationship, in order to improve slab quality, the use of PLC automatic control technology, while the use of sensors to gather real-time status of the slab, and collect data to the PLC, and then on the slab quality real-time monitoring, improve the automation level of continuous casting process, continuous casting production efficiency and economic benefits.
\end{abstract}

\section{Introduction}

The basic way of liquid steel molding including molding method and Two continuous casting method in which molding method to the production process between continuous features into the molten steel poured into the ingot mold, let it solidified into ingots and then processed into a desired billet size, however, after obtaining the continuous casting method as a new process is to cast molten steel through the continuous casting machine, condensation, slab cutting processes directly, as opposed to the traditional mold casting method, continuous casting steel casting technology not only speeds steel production efficiency and reduce the work intensity of workers, but also in large part to enhance the quality of the slab and metal unit time yield, while also significantly reducing power consumption and production cost.

In recent years, automatic secondary cooling water distribution, mold level prosecutions, tundish continuous temperature measurement techniques have been applied to many successive casting machines, but few chosen as steel slag stream detection technology, mold heat flux monitoring technologies and breakout prediction, slab quality tracking and determination technology. So, how to improve the current level of automation and quality slab continuous casting machine, speed up the production of metallurgical process efficiency, reduce production costs is one of China's steel enterprises cannot be ignored.

\section{System Design}

Caster mainly by the large package and tundish, mold and vibration device, the secondary cooling device, straightening device and a cutting device and other components, its production process shown in Figure 1. 


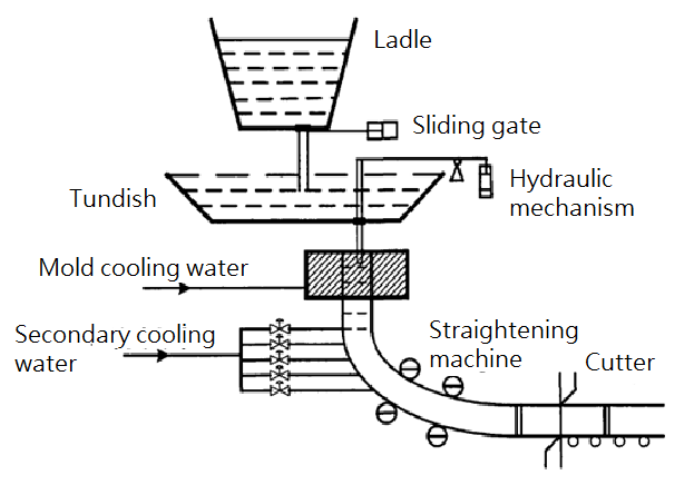

Fig. 1 continuous casting process

In recent years, in order to improve the quality of the slab in ensuring the efficiency of the premise of the continuous casting machine control systems are increasingly high requirements. Aiming at different production sites as well as the production environment control requirements, the design of continuous casting machine automatic control system into the management, monitoring and control layer, layer three levels. Among them, the macro-control management to achieve continuous casting process, the completion of the production data of the statistical summary of a variety of production and generate reports; monitoring level using the process control computer to complete the setting of production information monitoring and parameters, data storage, and communication and information management at the completion of the exchange of information; PLC control layer using on-site equipment to achieve real-time data acquisition and control information casting process. Casting automatic control system block diagram shown in Figure 2.

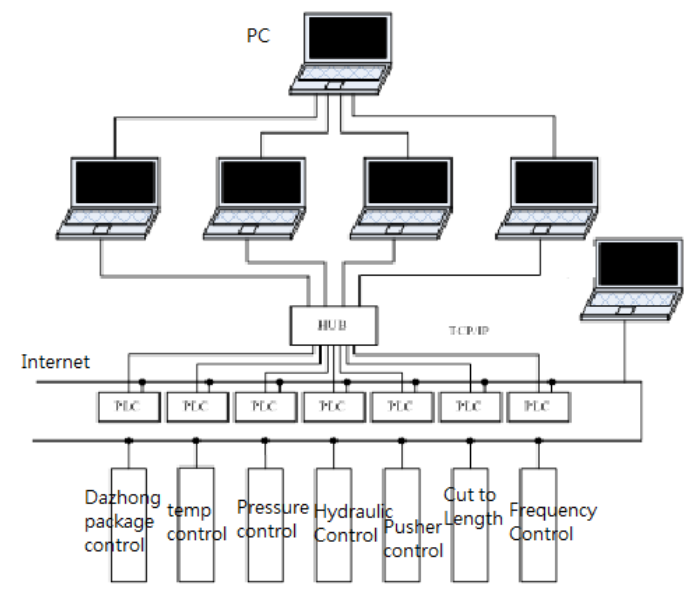

Fig. 2 Block diagram of continuous casting machine automatic control system

\section{Hardware Design}

Ladle turret having a rotary lifting, weighing and locking functions simultaneously carry two devices implement ladle casting operation. When casting ladle turret force is relatively large, the longer the period of rotation which produces the greater volatility, so not only to ensure the rotation control ladle turret precise smooth, but also to minimize the rotation time, so tundish level of volatility. Rotating selection of workers ladle turret frequency electrical control its position using pulse encoder is detected and the detected position signal is transmitted to the PLC, and finally after computing the angle of rotation of the rotary table and then determine its position. A place close to the ladle turret run a sync switch to achieve clearing pulse. When the driving power supply fails, the 
use of the manual valve to control the hydraulic drive, so ladle turret is rotated by the casting position to the accident location, in the process all the signal chain will be invalidated. When the ladle turret rotated to the casting position, take the disc brake lock-up mode, the use of PLC control coil KM11, KM12 and KM13 to achieve power through the motor and disc brake operation control. The electrical control shown in Figure 3.

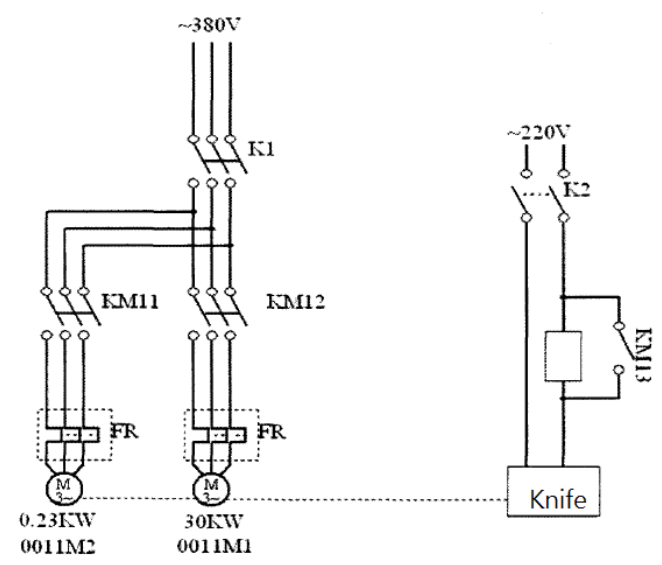

Fig. 3 Ladle turret electric control diagram

After sliding nozzle molten steel flow into the big bag tundish crucial level in the package molten steel, molten steel is too high will result in an overflow lead to accidents, resulting in too small so that the static pressure casting shell gap or discontinuity, the sliding gate opening and closing state control is extremely important. The system uses a hydraulic servo control, and the choice of the eddy current sensor level, including manual and automatic control mode. In manual mode, designed to direct the opening and closing as well as accident closed three operating modes; In automatic mode, set the molten steel surface by man-machine interface, and in the middle of the eddy current sensor device package level, and then the detection signal is fed back to the PLC, and finally calculated after the sliding movement of the output pack large amount of outlet, sliding gate control process shown in Figure 4.

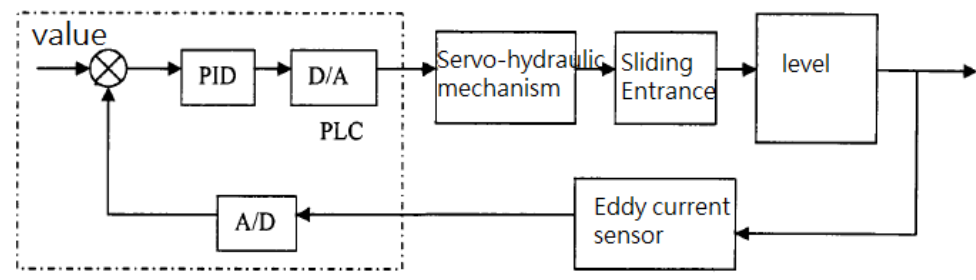

Fig. 4 large bag sliding gate control chart

The system uses electro-hydraulic servo system, hydraulic vibration equipment to achieve operational control of the turret and the car as well as the ups and downs of the mold, the design structure diagram shown in Figure 5. 


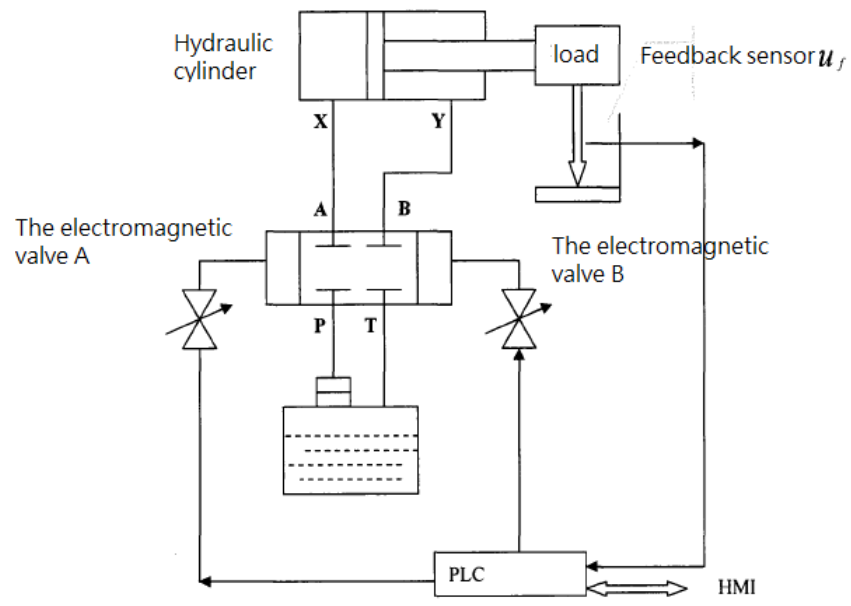

Fig. 5 Electro-hydraulic servo-hydraulic control chart

Operator send the corresponding control command by PLC, drive the hydraulic cylinder piston hydraulic push to complete the different levels of propulsion operations. Solenoid valves A, B when de-energized state, because the two are equal and the piston chamber pressure in a stationary state, that is, the middle of the solenoid valve; solenoid A or B is powered from two directions to complete the conversion of oil. Install the appropriate sound and light alarm devices, by pressure, voltage, respectively, tubing leaks, unstable power supply voltage abnormality monitoring, when monitoring data deviates from the normal 10\%, automatic alarm; installation of the heater and the cooling device, through the heat resistance temperature detector to achieve control.

Mold cooling water control includes a flow rate control, pressure control and temperature control in three aspects. Design of open water circulation system, using pumps pressurizing the cooling water to the crystallizer for its use, used to transport backwater rely residual pressure mechanical draft cooling tower cooling treatment, then returned to the suction well \# 1 , followed by recycling. The cooling water flow rate was set at $6 \sim 10 \mathrm{~m} / \mathrm{s}$, pressure was set to $1.2 \mathrm{MPa}$, and set the pressure, temperature, flow, and other detection devices, when the pressure is less than 0.8MP, two alarm is triggered, and when the pressure is less than $0.3 \mathrm{MP}$ when an alarm is triggered. Its mold cooling water control structure shown in Figure 6.

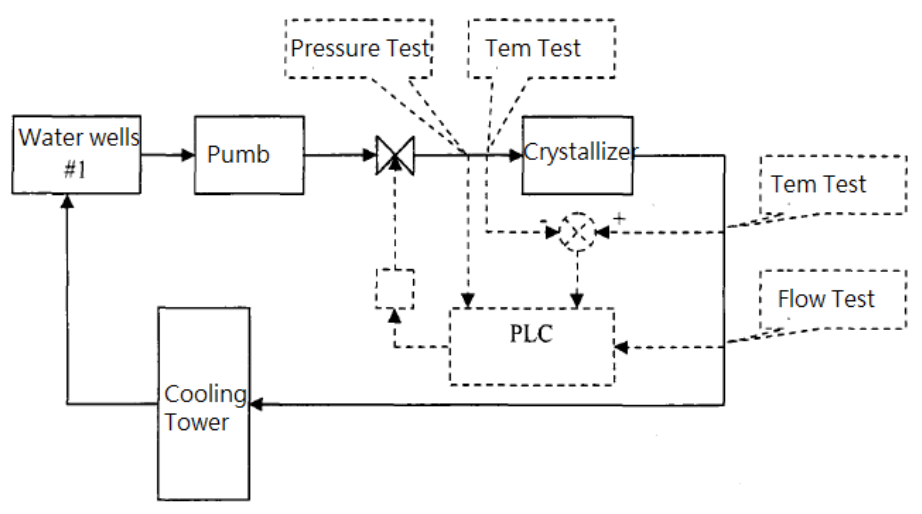

Fig. 6 Mold cooling water control structure diagram

Crystalline form of hydraulic vibration control device comprises a manual check and automatic three. Wherein, in manual mode, you can manually adjust and complete a variety of tests in inspection mode, intelligent detection can complete mold narrow faces taper, and in automatic 
mode, you can complete the normal casting Caster. Selected balance spring hinges and a combination of fixed gantry support structure so as to avoid vibration bar off phenomenon occurred in the course of the campaign; install two hydraulic cylinders with proportional valve control on both sides of the mold, so that mold may follow fast lifting cylinder quickly up and down vibration. In order to obtain a reliable hydraulic cylinder and the amplitude of the vibration displacement signal, the hydraulic cylinder device position sensor, and can effectively monitor the vibration state of each position by the hydraulic cylinder between the feedback signal of the position sensor, vibration controller according to the feedback signal to control the position sensor servo proportional valve opening, to achieve its coordination; eligible to mold the slab with friction due to vibration signal generated in the hydraulic cylinders on both sides of the pressure sensor device. Crystallizer vibration control system shown in Figure 7.

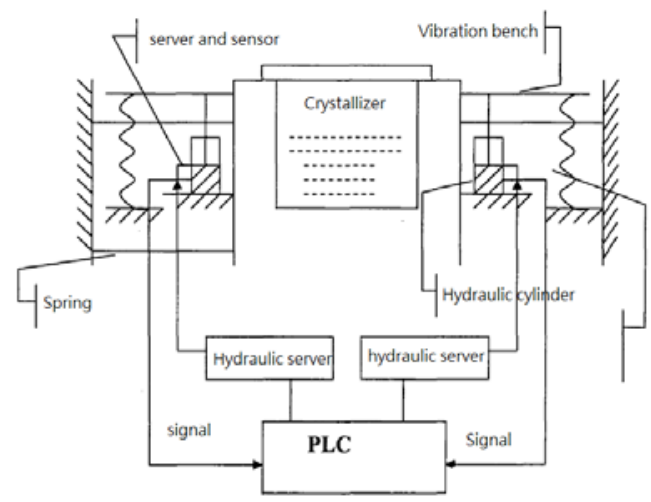

Fig. 7 Crystalline vibration control system of FIG

\section{Conclusion}

For recent years, the level of automation of the continuous casting machine control increasingly demanding problems, the use of continuous casting machine PLC precise control, combined with sensors slab running status real-time monitoring, automated control caster , greatly improve the level of automation of continuous casting and continuous casting process productivity, to give a higher economic efficiency.

\section{REFERENCE:}

[1] Zhang Guitao, Zhang Wenxia. Application of continuous casting machine computer control system [J]. Metallurgical Industry Automation, 2002, 06: 58-59.

[2] Da Xianjin. Design PLC control horizontal roller centrifugal casting machine [J]. Foundry Technology, 2014, 05: 1085-1087.

[3] Lei Yong, Wu Xiaobing. Maglev Centrifugal Casting Machine Micro Table Control Strategy [J]. Foundry Technology, 2013, 07: 927-929.

[4] Guo Hongquan, Chen Yuguo. Design and Implementation of Control System in Continuous Casting Machine Based on PLC [J]. Foundry Technology, 2015, 05: 1279-1280.

[5] Zhao Wenfeng. Control System Design and Simulation [M]. Xi'an: Xi'an University of Electronic Science and Technology Publishing, 2002.

[6] Tian Yanxiang editor. Casting Modern New Technology and New Technology and Slab Quality Control [M]. Beijing: Contemporary China Publishing House .2005.

[7] Liu Yuling. The Application of SIMATIC NET Technology in Continuous Casting Machine Control System [J]. Fluid Power Transmission and Control, 2007, 04: 33-35. 
[8] Shi Zhengxi,Zhang Zheng. Automatic control systems of Continuum-casting Machine Square-billet Caster [J]. Anhui Vocational College of Metallurgy and Technology, 2005,02: $48-49+57$. 